\title{
Metasequents and Tetravaluations
}

\author{
Rohan French $^{1}$
}

Received: 16 June 2020 / Accepted: 9 July 2021 / Published online: 25 August 2021

(C) The Author(s) 2021

\begin{abstract}
In this paper we treat metasequents-objects which stand to sequents as sequents stand to formulas - as first class logical citizens. To this end we provide a metasequent calculus, a sequent calculus which allows us to directly manipulate metasequents. We show that the various metasequent calculi we consider are sound and complete w.r.t. appropriate classes of tetravaluations where validity is understood locally. Finally we use our metasequent calculus to give direct syntactic proofs of various collapse results, closing a problem left open in French (Ergo, 3(5), 113-131 2016).
\end{abstract}

Keywords Metainferences · Nonreflexive logic $\cdot$ Nontransitive logic $\cdot$ Translations

\section{Introduction}

It is well known, by this stage, that for a great many tasks which we may be concerned with as logicians, conceiving of logics as merely sets of formulas-the logical truths according to that logic_-is inadequate. From a motivational point of view, Dummett points out that a focus on logical truths can have a distorting influence, and 'tempt us to regard logic as a search for logical truths' [8, p.168]. From a purely formal point of view, limiting ourselves to this framework results in an seemingly improper individuation of logics. Adapting some terminology from [19, p.103] let us call a policy on what to regard as the proper objects of study - the objects which we take a logic to be a set of-a logical framework. So, for example, we can call the conception on which a logic is merely a set of formulas FMLA, and the more familiar framework where a logic is a set of inferences from a set of premises to a conclusion the framework SET-FMLA, and the slightly less familiar framework where a logic is a set of multiple-conclusion inferences SET-SET. The problem about individuation mentioned above is simply the problem that there are a great many logics which are

Rohan French

rohan.french@gmail.com

1 Department of Philosophy, University of California, Davis, Davis, CA, 95616, USA 
identical in the framework FMLA, but distinct in frameworks like SET-FMLA and SETSET. For example, the logic of paradox has the same logical truths as classical logic ([23, p.228]) and so is the same logic in the framework FMLA, but differs from classical logic on which inferences it validates (and so is distinct from classical logic in the framework SET-FMLA). Similarly, there can be logics which are identical in the SET-FMLA framework, but distinct in the SET-SET framework. ${ }^{1}$

As noted in [19, p.190] the move from the FMLA to SET-FMLA and SET-SET frameworks is a step in the right direction, but there are nonetheless still many purposes for which we need to attend to more than simply which sequents or formulas are validated by a given logic. In particular, sometimes we need to move from considering which sequents are validated by a given logic to which sequent-to-sequent transitions, or as we will call them which metainferences or metasequents are validated by a given logic. One such purpose, of particular relevance to the present paper, is that of understanding substructural solutions to the paradoxes of self-reference. These are solutions which are characterized by their rejection of certain metasequents, ${ }^{2}$ and can result in logics which (depending on the logical vocabulary involved) can only be distinguished from classical logic at the level of valid metasequents and not at the level of valid sequents (see [26]).

Thinking of logics in terms of metasequents has also been put to philosophical use. For example, in [13] metasequents are used to explain how nonreflexive logics can "recapture full classical reasoning as being enthymematic" [13, p.123], and in [7] it is argued that the best way of understanding the inferentialist idea that the meaning of a logical constant is given by the rules which govern it is best understood when thinking of logics in terms of their derivable metasequents, rather than merely their derivable sequents.

The goal of the present paper is to take some initial steps in treating metasequents as first-class logical citizens. To this end we will focus in particular on the role of metasequents in proof, providing an explicit 'metasequent calculus' the rules of which manipulate metasequents directly. For the sake of simplicity we will focus on metasequent calculi for nonreflexive and nontransitive logics, allowing us to build on work done in [14] and [15] on the abstract valuational semantics for such logics in order to show that our metasequent calculi are sound and complete w.r.t. what is known in the literature on metainferences as 'local validity'. Finally we will then use our metasequent calculi to provide purely syntactic proofs of various 'collapse' results which have been given in the literature, e.g. in [6] and [2], which purport to

\footnotetext{
${ }^{1}$ One particularly interesting example involves the SET-SET consequence relations defined over Kripke models for intuitionistic logic, and Beth models for intuitionistic logic, so that $[\Gamma \succ \Delta]$ is Kripke-valid (resp. Beth-valid) iff for every point generated intuitionistic Kripke model $\mathcal{M}$ (respectively point generated Beth model $\mathcal{M}$ ) if every formula in $\Gamma$ holds at the generating point in $\mathcal{M}$, then some formula in $\Delta$ holds at the generating point. As is pointed out in $[16, p .28]$ both of these SET-SET consequence relations agree on SET-FMLA arguments-validating precisely the intuitionistically valid SET-FMLA arguments.

${ }^{2}$ This does not mean that more familiar paraconsistent and paracomplete logics do not involve the rejection of certain classically valid metasequents. For example, as pointed out in [4, p.851], typical paraconsistent logics end up rejecting the metasequent corresponding to the deduction theorem (which we will later write as $[\Gamma, A \succ B, \Delta] \Rightarrow[\Gamma \succ A \rightarrow B, \Delta])$, and typical paracomplete solutions end up rejecting the metasequent corresponding to proof by cases $([\Gamma, A \succ \Delta],[\Gamma, B \succ \Delta] \Rightarrow[\Gamma \succ A \vee B, \Delta])$.
} 
show that nontransitive logics are really just paraconsistent logics, showing that a similar connection can also be made between nonreflexive logics and paracomplete logics, settling in the affirmative a question left open in [13].

\section{The Metasequent Calculus}

Before we go on to introduce our metasequent calculus let us first settle some important notational and terminological issues concerning metainferences. Throughout we will work with a propositional language $\mathcal{L}$, the formulas of which are constructed out of a denumerably infinite supply of propositional variables $p_{0}, p_{1}, p_{2}, \ldots$ (the first three of which we abbreviated to $p, q, r$ ) using the binary connectives $\wedge$ ('and'), $\vee$ ('or'), and unary connective $\neg$ ( 'not'). For the majority of the paper we will work with sequents in the MSET-MSET framework, a (finitary) sequent for us being an ordered pair $\langle\Gamma, \Delta\rangle$ of finite multisets of formulas from $\mathcal{L}$. We will write such sequents as $[\Gamma \succ \Delta]$, with $\succ$ acting as our sequent separator. A (finitary) metasequent is an ordered pair $\langle\Sigma, \sigma\rangle$ consisting of a finite multiset $\Sigma$ of sequents, and a single sequent $\sigma$. We will write such a metasequent as $\Sigma \Rightarrow \sigma$.

Our metasequent calculi are modelled after hyperpersequent proof systems, introduced in [1], as a way of giving novel and tractable proof systems to various non-classical logics. ${ }^{3}$ Like hypersequent calculi the rules of our metasequent calculus are split into three kinds of rules: structural rules which govern metasequents as a whole, which we will call Outer Structural Rules, structural rules which govern the structural manipulation of sequents, which we will call Inner Structural Rules, and rules which introduce or eliminate connectives, our Operational Rules. In the majority of this paper we will be concerned with a variety of calculi which differ on what inner structural rules are present, but before introducing them it will be illustrative to explore some of the fine structure and design choices available in constructing metasequent calculi. To that end let us begin by introducing the main kinds of rules we will be concerned with.

OUter StRuctural Rules

$$
\begin{aligned}
& \overline{\sigma \Rightarrow \sigma}(I D) \quad \frac{\Sigma \Rightarrow \sigma}{\Sigma, \Sigma^{\prime} \Rightarrow \sigma}(D I L) \\
& \frac{\Sigma \Rightarrow \sigma \quad \sigma, \Sigma^{\prime} \Rightarrow \sigma^{\prime}}{\Sigma, \Sigma^{\prime} \Rightarrow \sigma^{\prime}}(\text { CUT) } \\
& \frac{\Sigma, \sigma^{\prime}, \sigma^{\prime} \Rightarrow \sigma}{\Sigma, \sigma^{\prime} \Rightarrow \sigma}(\text { CONTR })
\end{aligned}
$$

Readers familiar with the sequent calculus will recognize the outer structural rules of $(I D),(D I L),(C U T)$, and $(C O N T R)$ as being the sequent-language versions of

\footnotetext{
${ }^{3}$ In a similar way, one can see the calculus for metainferences introduced in [5] as being analogous to the tagged-formula calculi for modal logic introduced in [21]. The dual two-sided sequent calculi of [11] give a further, different again, way of using decorated sequent structures to give proof theory for nonreflexive and nontransitive logics with a very similar aim in mind. We leave further investigation of the connections between the present system and Fjellstad's to a future occasion.
} 
the familiar structural rules of identity or structural reflexivity, dilution or weakening, cut or transitivity, and contraction.

INNER STRUCTURAL RULES

$$
\begin{aligned}
& \frac{\Sigma \Rightarrow[X \succ Y]}{\Sigma \Rightarrow\left[X, X^{\prime} \succ Y, Y^{\prime}\right]}(D i l) \\
& \frac{\Sigma,[X \succ Y] \Rightarrow \sigma \quad \Sigma^{\prime},\left[X^{\prime} \succ Y^{\prime}\right] \Rightarrow \sigma}{\Sigma, \Sigma^{\prime},\left[X, X^{\prime} \succ Y, Y^{\prime}\right] \Rightarrow \sigma}(\text { CASES }) \\
& \frac{\Sigma \Rightarrow[X \succ Y, A, A]}{\Sigma \Rightarrow[X \succ Y, A]}(\text { ContrR }) c \quad \frac{\Sigma \Rightarrow[X, A, A \succ Y]}{\Sigma \Rightarrow[X, A \succ Y]}(\text { Contr } L) c \\
& \frac{\Sigma,[X \succ Y, A, A] \Rightarrow \sigma}{\Sigma,[X \succ Y, A] \Rightarrow \sigma}(\text { Contr } R) p \quad \frac{\Sigma,[X, A, A \succ Y] \Rightarrow \sigma}{\Sigma,[X, A \succ Y] \Rightarrow \sigma}(\text { Contr } L) p
\end{aligned}
$$

The rules here, along with the additional rules listed in Fig. 1, make up all the familiar structural rules (with the exception of permutation, which is invisible due to the use of multisets instead of sequences). The glaring exception to this, of course, is the rule of $(C A S E S)$. The rule of ( $C A S E S)$ is a 'safe' version of premise weakening, being essentially a metasequential formulation of the rule distinctive rule, called $(O r)$, of the nonmonotonic preferential logics in [20, p.190]. To see why we need a restricted version of weakening in the premises observe that the straightforward version of inner premise weakening, the rule which takes us from $\Sigma,[X>Y] \Rightarrow \sigma$ to $\Sigma,\left[X, X^{\prime} \succ Y, Y^{\prime}\right] \Rightarrow \sigma$ allows for the following derivation:

$$
\frac{\overline{[\succ] \Rightarrow[\succ]}_{[\succ] \Rightarrow[X \succ Y]}^{(I D)}}{(\text { Dil })}
$$

which says that any sequent has as a metainferential consequence any other sequent, rendering the relation of metainferential consequence trivial. Similarly, given the presence of $(D i l)$ restricting this rule in an analogous way to the rule of cautious monotonocity, where we'd essentially add an additional premise that we must have $[X \succ Y] \Rightarrow\left[X^{\prime} \succ Y^{\prime}\right]$, will also allow for the same derivation as the requisite premise is derivable using $(I D)$ and $(D i l)$. For more on the formulation of (CASES) see also the brief discussion after Theorem 1 below.

Unlike most hypersequent calculi, where the different sequent components are all understood to be on a par, our metasequent calculi treats our premise sequents, and our conclusion sequent slightly differently. To that end we have two different families of operational rules - those where the active sequent(s) occur in conclusion position, and those where the active sequent(s) occur in premise position. We will also have two different sub-families within these two families-introduction rules, which introduce a complex formula on the left or the right of a given sequent, and their inverses, elimination rules, which eliminate a given complex formula on the left or the right of a given sequent.

Conclusion Active Operational Rules

Introduction Rules

$$
\frac{\Sigma \Rightarrow[X \succ Y, A] \quad \Sigma \Rightarrow[X \succ Y, B]}{\Sigma \Rightarrow[X \succ Y, A \wedge B]}(\wedge R) c
$$




$$
\begin{gathered}
\frac{\Sigma \Rightarrow[X, A, B \succ Y]}{\Sigma \Rightarrow[X, A \wedge B \succ Y]}(\wedge L) c \\
\frac{\Sigma \Rightarrow[X \succ Y, A, B]}{\Sigma \Rightarrow[X \succ Y, A \vee B]}(\vee R) c \\
\frac{\Sigma \Rightarrow[X, A \succ Y] \quad \Sigma \Rightarrow[X, B \succ Y]}{\Sigma \Rightarrow[X, A \vee B \succ Y]}(\vee L) c \\
\frac{\Sigma \Rightarrow[X, A \succ Y]}{\Sigma \Rightarrow[X \succ Y, \neg A]}(\neg R) c \quad \frac{\Sigma \Rightarrow[X \succ Y, A]}{\Sigma \Rightarrow[X, \neg A \succ Y]}(\neg L) c
\end{gathered}
$$

Elimination Rules

$$
\begin{gathered}
\frac{\Sigma \Rightarrow[X \succ Y, A \wedge B]}{\Sigma \Rightarrow[X \succ Y, A]}(\wedge R) c \uparrow 1 \quad \frac{\Sigma \Rightarrow[X \succ Y, A \wedge B]}{\Sigma \Rightarrow[X \succ Y, B]}(\wedge R) c \uparrow 2 \\
\frac{\Sigma \Rightarrow[X, A \wedge B \succ Y]}{\Sigma \Rightarrow[X, A, B \succ Y]}(\wedge L) c \uparrow \\
\frac{\Sigma \Rightarrow[X \succ Y, A \vee B]}{\Sigma \Rightarrow[X \succ Y, A, B]}(\vee R) c \uparrow \\
\frac{\Sigma \Rightarrow[X, A \vee B \succ Y]}{\Sigma \Rightarrow[X, A \succ Y]}(\vee L) c \uparrow 1 \quad \frac{\Sigma \Rightarrow[X, A \vee B \succ Y]}{\Sigma \Rightarrow[X, B \succ Y]}(\vee L) c \uparrow 2 \\
\frac{\Sigma \Rightarrow[X \succ Y, \neg A]}{\Sigma \Rightarrow[X, A \succ Y]}(\neg R) c \uparrow \quad \frac{\Sigma \Rightarrow[X, \neg A \succ Y]}{\Sigma \Rightarrow[X \succ Y, A]}(\neg L) c \uparrow
\end{gathered}
$$

Premise ACtive Operational Rules

Introduction Rules

$$
\begin{aligned}
& \frac{\Sigma,[X \succ Y, A],[X \succ Y, B] \Rightarrow \sigma}{\Sigma,[X \succ Y, A \wedge B] \Rightarrow \sigma}(\wedge R) p \quad \frac{\Sigma,[X, A, B \succ Y] \Rightarrow \sigma}{\Sigma,[X, A \wedge B \succ Y] \Rightarrow \sigma}(\wedge L) p \\
& \frac{\Sigma,[X \succ Y, A, B] \Rightarrow \sigma}{\Sigma,[X \succ Y, A \vee B] \Rightarrow \sigma}(\vee R) p \quad \frac{\Sigma,[X, A \succ Y],[X, B \succ Y] \Rightarrow \sigma}{\Sigma,[X, A \vee B \succ Y] \Rightarrow \sigma}(\vee L) p \\
& \frac{\Sigma,[X, A \succ Y] \Rightarrow \sigma}{\Sigma,[X \succ Y, \neg A] \Rightarrow \sigma}(\neg R) p \quad \frac{\Sigma,[X \succ Y, A] \Rightarrow \sigma}{\Sigma,[X, \neg A \succ Y] \Rightarrow \sigma}(\neg L) p
\end{aligned}
$$

\section{Elimination Rules}

$$
\begin{array}{cc}
\frac{\Sigma,[X \succ Y, A \wedge B] \Rightarrow \sigma}{\Sigma,[X \succ Y, A],[X \succ Y, B] \Rightarrow \sigma}(\wedge R) p \uparrow & \frac{\Sigma,[X, A \wedge B \succ Y] \Rightarrow \sigma}{\Sigma,[X, A, B \succ Y] \Rightarrow \sigma}(\wedge L) p \uparrow \\
\frac{\Sigma,[X \succ Y, A \vee B] \Rightarrow \sigma}{\Sigma,[X \succ Y, A, B] \Rightarrow \sigma}(\vee R) p \uparrow & \frac{\Sigma,[X, A \vee B \succ Y] \Rightarrow \sigma}{\Sigma,[X, A \succ Y],[X, B \succ Y] \Rightarrow \sigma}(\vee L) p \uparrow \\
\frac{\Sigma,[X \succ Y, \neg A] \Rightarrow \sigma}{\Sigma,[X, A \succ Y] \Rightarrow \sigma}(\neg R) p \uparrow & \frac{\Sigma,[X, \neg A \succ Y] \Rightarrow \sigma}{\Sigma,[X \succ Y, A] \Rightarrow \sigma}(\neg L) p \uparrow
\end{array}
$$

Throughout this paper we will be interested in exploring a number of different metasequent calculi, and so it will be helpful to lay out a map of some of the relevant proof-theoretic terrain. To this end let us define the following families of metasequent calculi.

Firstly our basic metasequent calculi is the system S consisting solely of the inner and outer structural rules above. There are a variety of different ways of constructing other metasequent calculi from our basic system by either adding or removing 
certain rules from $S$ as described below. Let $X$ be a metasequent calculi, then the metasequent calculi

- $\quad X *$ is the result of removing (CUT) from $X$

- $\quad X+$ is the result of removing (CASES) from $X$

- $\quad X p I$ is the result of adding the premise active introduction rules to $X$

- $X p E$ is the result of adding the premise active elimination rules to $X$

- $X c I$ is the result of adding the conclusion active introduction rules to $X$

- $X c E$ is the result of adding the conclusion active elimination rules to $X$

- $\quad X \mathrm{R}$ is the result of adding the initial sequent $(I d)$ from Fig. 1 to $X$

- $\quad X \mathrm{~T}$ is the result of adding the rule $(\mathrm{Cut})$ Fig. 1 to $X$

Our basic metasequent calculi will be the system SpIcI, which we will dub $\mathbb{M}$. In the remainder of this section we will chart out the following relationships between various different metasequent calculi.

- Inner Contraction is derivable from the outer structural rules, $(C A S E S)$, and (Dil) (Theorem 1)

- The rule of $(I D)$ can be restricted to sequents containing only a single propositional variable without a loss of any $\mathbb{M}$-derivable metasequents (Theorem 2)

- The premise active introduction rules are derivable in $\mathrm{S} c E$ (Theorem 3)

- The conclusion active elimination rules are derivable in $S p I$ (Theorem 4)

- The premise active elimination rules are derivable in $S c I$ (Theorem 5)

- The conclusion active introduction rules are derivable in $\mathrm{S} p E$ (Theorem 6)

Theorem 1 (Derivability of Inner Contraction) The rules of conclusion active contraction

$$
\frac{\Sigma \Rightarrow[X \succ Y, A, A]}{\Sigma \Rightarrow[X \succ Y, A]}(\text { Contr } R) c \quad \frac{\Sigma \Rightarrow[X, A, A \succ Y]}{\Sigma \Rightarrow[X, A \succ Y]}(\text { Contr } L) c
$$

and premise active contraction

$$
\frac{\Sigma,[X \succ Y, A, A] \Rightarrow \sigma}{\Sigma,[X \succ Y, A] \Rightarrow \sigma}(\text { Contr }) p \quad \frac{\Sigma,[X, A, A \succ Y] \Rightarrow \sigma}{\Sigma,[X, A \succ Y] \Rightarrow \sigma}(\operatorname{Contr} L) p
$$

are derivable from the outer structural rules, $(C A S E S)$ and (Dil).

$$
\begin{gathered}
\overline{\Sigma \Rightarrow[A \succ A]}(I d) \\
\frac{\Sigma \Rightarrow[X \succ Y, A] \quad \Sigma^{\prime} \Rightarrow\left[A, X^{\prime} \succ Y^{\prime}\right]}{\Sigma, \Sigma^{\prime} \Rightarrow\left[X, X^{\prime} \succ Y, Y^{\prime}\right]}(C u t)
\end{gathered}
$$

Fig. 1 Additional Inner Structural Rules for $X \mathrm{R}$ and $X \mathrm{~T}$ systems 
Proof We give the derivation for $($ Contr $R) c$ and $($ ContrL $L$, the ones for $($ Contr $L) c$ and $($ Contr $R) p$ being similar.

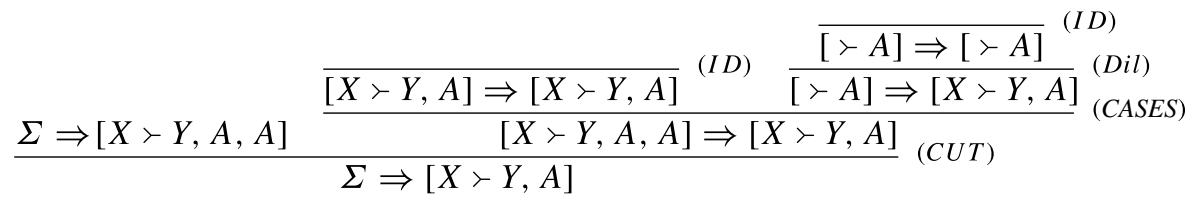

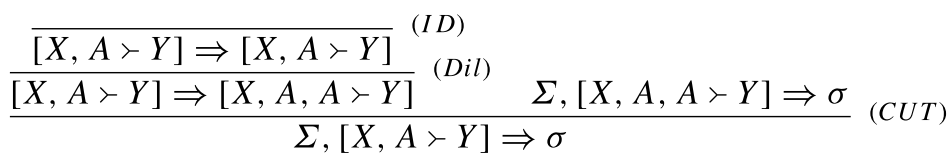

The above result has some prima facie similarities to the dilemma noncontractivists face regarding cautious cut, as elaborated in [27]. Like cautious cut (CASES) is a safe version of a rule which the noncontractivist should want-in this case ( $C A S E S$ ) is a safe version of premise weakening. Unlike the case of cautious cut, though, the affine contractivist can weaken $(C A S E S)$ while still retaining many of its uses by thinking of the relevant kind of sequent combination which occurs in the application of ( $C A S E S$ ) as not involving multiset addition, as we have it here, but rather as involving multiset union proper, where $[A] \cup[A]$ is just $[A]$ and $\operatorname{not}[A, A]{ }^{4}$ This gives the affine contractivist a way of avoiding the derivation of the conclusion contraction rules.

Theorem 2 Let $\mathbb{M}-$ be $\mathbb{M}$ with (I D) replaced by the structural rules

$$
(I D) l-\quad \overline{[p \succ] \Rightarrow[p \succ]} \quad(I D) r-\quad \overline{[\succ p] \Rightarrow[\succ p]}
$$

Then (I D) is derivable in $\mathbb{M}$-.

Proof Our proof proceeds in two stages. Firstly we will show that from $(I D) l-$ and $(I D) r$ - we can derive all instances of $(I D)$ where $\sigma=[A \succ]$ and $\sigma=[\succ A]$. Our proof of this proceeds by induction on the complexity of $A$, the basis case is given by $(I D) l-$ and $(I D) r$ - themselves. Our induction hypothesis yields that we can derive the instances of $(I D)$ where $\sigma=[B \succ]$ and $\sigma=[\succ B]$ where the complexity of $B$ is $n$. The cases in the induction step are simple and familiar, we give those for $\sigma=[\neg B \succ]$ and $\sigma=\left[\succ B_{1} \wedge B_{2}\right]$. The derivations we need are below

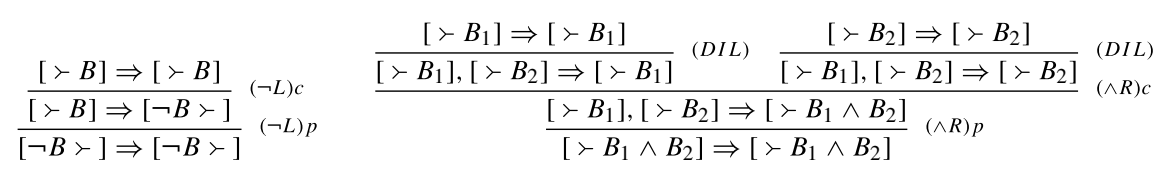

By the induction hypothesis the leaf sequents in the above two derivations can be derived in $\mathbb{M}-$, and so it follows that all instances of $(I D)$ where $\sigma=[A \succ]$ and $\sigma=[\succ A]$ are derivable in $\mathbb{M}-$.

\footnotetext{
${ }^{4}$ For more on the distinctions between different operations on multisets see [30, p.42f]. For a clear example of how noncontractive logics are sensitive to the distinction between these two different kinds of union operations see [28].
} 
The second stage of our proof simply involves showing that using $(C A S E S)$ and (Dil) we can show that if $[X>Y] \Rightarrow[X>Y]$ and $\left[X^{\prime}>Y^{\prime}\right] \Rightarrow\left[X^{\prime}>Y^{\prime}\right]$ are derivable then so is $\left[X, X^{\prime} \succ Y, Y^{\prime}\right] \Rightarrow\left[X, X^{\prime}>Y, Y^{\prime}\right]$.

$$
\frac{\frac{[X \succ Y] \Rightarrow[X \succ Y]}{[X \succ Y] \Rightarrow\left[X, X^{\prime} \succ Y, Y^{\prime}\right]}(\text { Dil }) \quad \frac{\left[X^{\prime} \succ Y^{\prime}\right] \Rightarrow\left[X^{\prime} \succ Y^{\prime}\right]}{\left[X^{\prime} \succ Y^{\prime}\right] \Rightarrow\left[X, X^{\prime} \succ Y, Y^{\prime}\right]}}{\left[X, X^{\prime} \succ Y, Y^{\prime}\right] \Rightarrow\left[X, X^{\prime} \succ Y, Y^{\prime}\right]} \text { (DASES) }
$$

So the metasequent $\left[A_{1}, \ldots, A_{n} \succ B_{1}, \ldots, B_{m}\right] \Rightarrow\left[A_{1}, \ldots, A_{n} \succ B_{1}, \ldots, B_{m}\right]$ can be derived in $\mathbb{M}-$ by repeated applications of this result to sequents of the form $\left[A_{i} \succ\right] \Rightarrow\left[A_{i} \succ\right]$ and $\left[\succ B_{i}\right] \Rightarrow\left[\succ B_{i}\right]$, which in the first part of our proof we proved were derivable in $\mathbb{M}-$.

In the above proof we can see a nice example of the way in which (CASES) acts in a very similar way to $(D i l)$. This proof does make extensive use of our rules $(D i l)$ and $(C A S E S)$, and so a different restriction on the sequents in $(I D)$ would be required to facilitate an admissibility proof for $(D i l)$ or $(C A S E S)$, such as requiring $\sigma$ to contain only propositional variables. The above result is primarily intended as an illustration of the proof-theoretic role played by $(C A S E S)$, and we leave the investigation of such other restrictions on $(I D)$ for future work.

The next four results are concerned with the connections between our premise and conclusion operational rules. In summary what they show is that we can trade off what we might think of as the 'polarity' of an operational rule (whether it introduces or eliminates a connective) against the position in the metainference in which it operates (on premise sequents or the conclusion sequent).

\section{Theorem 3 The premise active introduction rules are derivable in ScE}

Proof Suppose that $(R) c$ is a conclusion active introduction rule, and $(R) c \uparrow_{i}$ its corresponding conclusion active elimination rules, and $(R) p$ the premise active introduction rule corresponding to $(R) c$.

$$
\frac{\Sigma \Rightarrow \sigma_{1} \ldots \quad \Sigma \Rightarrow \sigma_{n}}{\Sigma \Rightarrow \sigma}(R) c \quad \frac{\Sigma \Rightarrow \sigma}{\Sigma \Rightarrow \sigma_{i}}(R) c \uparrow_{i} \quad \frac{\Sigma, \sigma_{1}, \ldots, \sigma_{n} \Rightarrow \sigma^{\prime}}{\Sigma, \sigma \Rightarrow \sigma^{\prime}}(R) p
$$

We show that $(R) p$ is derivable using $(R) c \uparrow_{i},(I D),(C U T)$ and $(C O N T R)$.

$$
\begin{aligned}
& \frac{\frac{\overline{\sigma \Rightarrow \sigma}_{\sigma \Rightarrow \sigma_{1}}}{{ }^{(I D) c \uparrow}{ }_{1} \quad \Sigma, \sigma_{1}, \sigma_{2}, \ldots, \sigma_{n} \Rightarrow \sigma^{\prime}}}{\Sigma, \sigma, \sigma_{2}, \ldots, \sigma_{n} \Rightarrow \sigma^{\prime}}(C U T)
\end{aligned}
$$

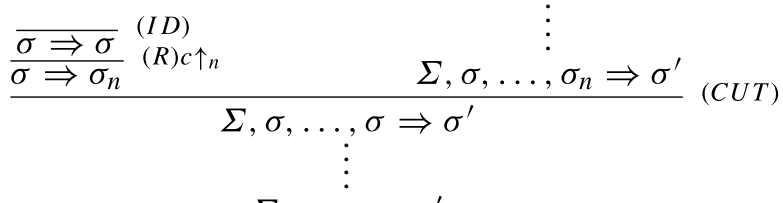

$$
\begin{aligned}
& \frac{\Sigma, \sigma, \sigma \Rightarrow \sigma^{\prime}}{\Sigma, \sigma \Rightarrow \sigma^{\prime}}(\text { CONTR })
\end{aligned}
$$

Theorem 4 The conclusion active elimination rules are derivable in SpI 
Proof Suppose that $(R) c$ is a conclusion active introduction rule, and $(R) c \uparrow_{i}$ its corresponding conclusion active elimination rules, and $(R) p$ the premise active introduction rule corresponding to $(R) c$.

$$
\frac{\Sigma \Rightarrow \sigma_{1} \ldots \quad \Sigma \Rightarrow \sigma_{n}}{\Sigma \Rightarrow \sigma}(R) c \quad \frac{\Sigma \Rightarrow \sigma}{\Sigma \Rightarrow \sigma_{i}}(R) c \uparrow_{i} \quad \frac{\Sigma, \sigma_{1}, \ldots, \sigma_{n} \Rightarrow \sigma^{\prime}}{\Sigma, \sigma \Rightarrow \sigma^{\prime}}(R) p
$$

We show that $(R) c \uparrow$ is derivable using $(R) p,(I D),(C U T)$ and $(D I L)$.

$$
\frac{\bar{\sigma}_{i} \Rightarrow \sigma_{i}}{(I D)}(\text { DIL) }
$$

Theorem 5 The premise active elimination rules are derivable in ScI

Proof Suppose that $(R) c$ is a conclusion active introduction rule, and $(R) c \uparrow_{i}$ its corresponding conclusion active elimination rules, and $(R) p \uparrow$ the premise active elimination rule corresponding to $(R) c$.

$$
\frac{\Sigma \Rightarrow \sigma_{1} \ldots \Sigma \Rightarrow \sigma_{n}}{\Sigma \Rightarrow \sigma}(R) c \quad \frac{\Sigma \Rightarrow \sigma}{\Sigma \Rightarrow \sigma_{i}}(R) c \uparrow_{i} \quad \frac{\Sigma, \sigma \Rightarrow \sigma^{\prime}}{\Sigma, \sigma_{1}, \ldots, \sigma_{n} \Rightarrow \sigma^{\prime}}(R) p \uparrow
$$

We show that $(R) p \uparrow$ is derivable using $(R) c,(I D),(C U T)$ and $(D I L)$.

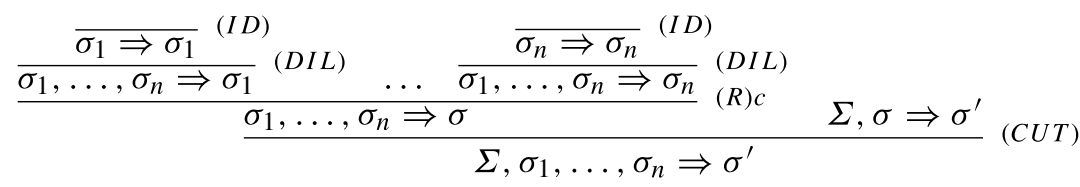

Theorem 6 The conclusion active introduction rules are derivable in $\mathrm{SpE}$

Proof Suppose that $(R) c$ is a conclusion active introduction rule, and $(R) c \uparrow_{i}$ its corresponding conclusion active elimination rules, and $(R) p \uparrow$ the premise active elimination rule corresponding to $(R) c$.

$$
\frac{\Sigma \Rightarrow \sigma_{1} \ldots \quad \Sigma \Rightarrow \sigma_{n}}{\Sigma \Rightarrow \sigma}(R) c \quad \frac{\Sigma \Rightarrow \sigma}{\Sigma \Rightarrow \sigma_{i}}(R) c \uparrow i \quad \frac{\Sigma, \sigma \Rightarrow \sigma^{\prime}}{\Sigma, \sigma_{1}, \ldots, \sigma_{n} \Rightarrow \sigma^{\prime}}(R) p \uparrow
$$

We show that $(R) c$ is derivable using $(R) p \uparrow,(I D),(C U T)$ and $(C O N T R)$.

$$
\begin{aligned}
& \frac{\Sigma \Rightarrow \sigma_{1} \quad \frac{\overline{\sigma \Rightarrow \sigma}{ }^{(I D)}}{\sigma_{1}, \ldots, \sigma_{n} \Rightarrow \sigma}}{(R) p \uparrow}(\text { (CUT) } \\
& \frac{\Sigma \Rightarrow \sigma_{n} \quad \Sigma, \ldots, \dot{\Sigma}, \sigma_{n} \Rightarrow \sigma}{\Sigma, \ldots, \Sigma, \sum \underset{(\text { CONTR })}{(C U T)}} \\
& \Sigma \stackrel{\Rightarrow}{\Rightarrow} \sigma
\end{aligned}
$$


What the above results tell us is that we can trade off premise operational rules and conclusion operational rules against one another. Of particular interest are Theorems 3 and 5 which show us how the premise operational rules follow from the conclusion introduction and elimination rules. Given that the premise operational rules are largely invisible unless we are manipulating metasequents directly, this gives us some insight into why the elimination operational rules have been so essential in reasoning about metainferences derivatively as sequent-to-sequent transitions. This goes some way to explaining why in work such as [13, p.121] and [6, p.394f], for example, there is an emphasis on the importance of the $c E$-rules. The systems under investigation there (essentially our $S c I c E R$ and $S c I c E T$ ), where metainferences are treated as proof-theoretically second-class citizens, are designed to capture a class of metainferences which includes various canonical 'elimination metainferences' like $[\succ A \wedge B] \Rightarrow[\succ A]$ which can only be derived using $c E$ rules. ${ }^{5}$ For example:

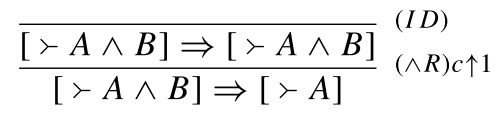

In systems like SpIcI, though, we can derive this using only inferences which increase the overall complexity of the metasequent.

$$
\frac{\overline{[\succ A] \Rightarrow[\succ A]}^{(\succ D)}}{[\succ A \wedge[\succ B] \Rightarrow[\succ A]}(D I L)
$$

For this reason in what follows we will primarily be focused on systems in the SpIcI-family. Throughout, given a metasequent calculus $X$, we will record the claim that a metasequent $\Sigma \Rightarrow \sigma$ is derivable in $X$ as $\vdash_{X} \quad \Sigma \Rightarrow \sigma$. So, for example, the above two derivations can be taken as showing that $\vdash_{S c E}[\succ A \wedge B] \Rightarrow[\succ A]$ and $\vdash_{S p I}[\succ A \wedge B] \Rightarrow[\succ A]$ respectively.

\section{Soundness \& Completeness}

\subsection{Tetravaluations}

What we will show in this section is that the metasequent calculi defined in the previous section appropriately capture local validity for a natural class of valuations. In particular we will make use of the work done in [14] and [15] (building off [18]) on what are called tetravaluations, functions from our propositional language $\mathcal{L}$ into the

\footnotetext{
${ }^{5}$ A more controversial and philosophically interesting example concerns the 'metarule of modus ponens', roughly the metainference represented by the metasequent $[\succ A \rightarrow B],[\succ A] \Rightarrow[\succ B]$, on which the interested reader should see [10] for a discussion of the relevant philosophical controversy.
} 
set of values $\{\top, \perp, 工, *\}{ }^{6}$ Throughout we will work with the following definition of what it is for a tetravaluation to be a counterexample to a sequent.

Definition 1 (Counterexample) A tetravaluation $v$ is a counterexample to a sequent $[\Gamma \succ \Delta]$-written $v \mid \forall[\Gamma \succ \Delta]$-iff $v[\Gamma] \subseteq\{\top, I\}$ and $v[\Delta] \subseteq\{\perp, I\} .{ }^{7}$ A sequent $[\Gamma \succ \Delta]$ holds on a tetravaluation $v$-written $v \Vdash[\Gamma \succ \Delta]$ iff $v$ isn't a counterexample to $[\Gamma>\Delta]$.

In our completeness proof we will make special use of a particular kind of counterexample that exists given an argument $[\Gamma \succ \Delta]$, namely its exact counterxample. Given sequents $[\Gamma \succ \Delta]$ and $\left[\Gamma^{\prime} \succ \Delta^{\prime}\right]$ let us say that $[\Gamma \succ \Delta] \sqsubseteq\left[\Gamma^{\prime} \succ \Delta^{\prime}\right]$ iff $\operatorname{Dom}(\Gamma) \subseteq \operatorname{Dom}\left(\Gamma^{\prime}\right)$ and $\operatorname{Dom}(\Delta) \subseteq \operatorname{Dom}\left(\Delta^{\prime}\right)$, where $\operatorname{Dom}(\Gamma)$, for $\Gamma$ a multiset, is the underlying (or ground) set of $\Gamma$. Then we have the following:

Definition 2 Given a sequent $a=[\Gamma \succ \Delta]$ the exact counterexample for $a$ is the tetravaluation $v_{a}$ where:

$$
v_{a}(A)= \begin{cases}\top & \text { iff } A \in \Gamma \backslash \Delta, \\ \perp & \text { iff } A \in \Delta \backslash \Gamma, \\ 工 & \text { iff } A \in \Gamma \cap \Delta, \\ * & \text { iff } A \notin \Gamma \cup \Delta .\end{cases}
$$

Proposition 1 For all sequents $a, b$, we have that $v_{a} \nvdash b$ iff $b \sqsubseteq a$.

The above definitions follow those given as Definition 9 and Proposition 8 in the appendix of [15]. As noted there, in [14] the notion of an exact counterexample is introduced as being a valuation which satisfies Proposition 1, the particular tetravaluation given in Definition 2 being used to show that they always exist. Foreshadowing a little, in our completeness proof we will construct the exact counterexample for a particular sequent which includes the conclusion sequent of our target invalid metasequent, and make use of Proposition 1 to ensure that it is a local counterexample to the whole metasequent.

Now, as it happens we will not be concerned with arbitrary tetravaluations here, for obvious reasons. In particular we want tetravaluations which will appropriately capture the truth-conditions of the connectives, and are suitably sensitive to which of the (internal) structural rules from Fig. 1 are present in our metasequent calculus.

To deal with the first of the above points we will exploit the particular structure which our set of values is typically taken to have. The set of values $\{\top, \perp, I, *\}$

\footnotetext{
${ }^{6}$ In those papers French and Ripley are concerned with various abstract features of the valuational semantics for nonreflexive and nontransitive logics. Our goals in the present paper will be much more targeted, our concern being to use tetravaluations to semantically interpret metainferences. There is definitely work to be done on looking at the abstract connections between metasequents and tetravaluations, some initial work in that direction appearing in [19, p.132-136]

${ }^{7}$ Here we deviate from the notation used in [14] and [15], writing ' $v \Downarrow \sigma$ ' for what there would be written as ' $v$ '.
} 
is taken to be a bilattice in [14] and [15], with two distinct lattice orders $\sqsubseteq$ (the information order), and $\preccurlyeq$ (the truth order). We will only be concerned with the truth ordering on values, here, according to which these four values are ordered as follows:

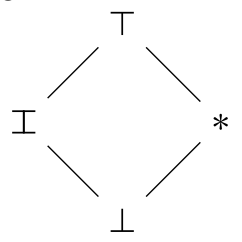

Definition 3 Say that a tetravaluation $v$ is truth-fitting iff

$-\quad v(A \wedge B)=v(A) \curlywedge v(B)$,

- $v(A \vee B)=v(A) \curlyvee v(B)$, and

$-v(\neg A)=\sim v(A)$.

where $\curlywedge$ and $\curlyvee$ are the (truth) meet and (truth) join in the above lattice, and $\sim$ is the operation on values for which $\sim \perp=\top, \sim \top=\perp$ and $\sim x=x$ otherwise.

Unsurprisingly, this interpretation of the connectives gives us matrices which are isomorphic to the four-valued matrices for the logic of first-degree entailment, the lattice of our information-ordering being the approximation lattice, and that of our truth-ordering being the logical lattice of [3]. We will return to the connections between the metainferential validity defined in terms of tetravaluations and the logic of first-degree entailment and its extensions in the next section.

Finally, in order to obtain the right connection between our classes of tetravaluations and what inner structural rules are present, we will also want to consider the following two more restrictive classes of tetravaluations.

Definition 4 Let $\mathfrak{V}$ be the class of all tetravaluations. Then:

- Let $\mathfrak{V}^{r}$ be the set of all tetravaluations $v$ where for no formula $A$ do we have $v(A)=I$. Call this the class of all reflexive tetravaluations

- Let $\mathfrak{V}^{t}$ be the set of all tetravaluations $v$ where for no formula $A$ do we have $v(A)=*$. Call this the class of all transitive tetravaluations.

The reason for focusing on these particular two classes of tetravaluations is given by the fact that $(I d)$ holds on all valuations in $\mathfrak{V}^{r}$, and $(C u t)$ preserves holding on all valuations in $\mathfrak{V}^{t}{ }^{8}$

Finally, we will lift the above definition of what it is for a tetravaluation to be a counterexample to a sequent to also cover metasequents. In particular our focus here will be on what is known in the literature as local validity for metainferences.

Definition 5 A valuation $v$ is a (local) counterexample to a metasequent $\Sigma \Rightarrow \sigma$ (which we will write as $v \Downarrow \Sigma \Rightarrow \sigma$ ) iff we have $v \Vdash \sigma^{\prime}$ for every $\sigma^{\prime} \in \Sigma$, and $v \Downarrow \sigma$.

${ }^{8}$ These two facts, put in a slightly different key, appear as part of Theorem 2 of [14, p.1322]. 
A metasequent holds on a valuation $v$ iff $v$ is not a counterexample to it, which we will write as $v \Vdash \Sigma \Rightarrow \sigma$

This notion of a local counterexample induces a corresponding local notion of validity for metasequents, as follows.

Definition 6 A metasequent $\Sigma \Rightarrow \sigma$ is locally valid relative to a set $V$ of tetravaluations iff it holds on every $v \in V$.

This contrasts with the following global notion of validity.

Definition 7 A metasequent $\Sigma \Rightarrow \sigma$ is globally valid relative to a set $V$ of tetravaluations iff if for every $V^{\prime} \subseteq V$, if for every $\sigma^{\prime} \in \Sigma, \sigma^{\prime}$ holds on every $v \in V^{\prime}$, then for every $V^{\prime} \subseteq V, \sigma$ holds on every $v \in V^{\prime}$.

So, for example, the metasequent $[\succ p] \Rightarrow[\succ q]$ will count as globally valid relative to the $\mathfrak{V}$ (as $[\succ p$ ] does not hold on any tetravaluation $v \in \mathfrak{V}$ for which $v(p)=$ $\perp$ ), but not locally valid relative to the same class of valuations (any tetravaluation $v \in \mathfrak{V}$ for which $v(p)=\top$ and $v(q)=\perp$ will be a local counterexample to this metasequent). ${ }^{9}$ The situation becomes significantly more complex if we are dealing with metainference schemas, as opposed to 'concrete' metainferences like we are concerned with in the present paper, though, for more on which the interested reader should see [31]. For the remainder of this paper we will solely be concerned with local counterexamples, and correspondingly with local validity.

\subsection{Soundness}

Theorem 7 (Soundness) If a metasequent $\Sigma \Rightarrow \sigma$ is derivable in $\mathbb{M}$ (resp. $\mathbb{M R}$, $\mathrm{MT})$ then that metasequent has no counterexample among the class of all truth-fitting tetravaluations (resp. truth-fitting reflexive tetravaluations, truth-fitting transitive tetravaluations).

Proof We prove the contrapositive-showing that if a truth-fitting tetravaluation (resp. truth-fitting reflexive tetravaluation, truth-fitting transitive tetravaluation) of the right kind is a counterexample to the metasequent $\Sigma \Rightarrow \sigma$ then that metasequent isn't derivable in $\mathbb{M}$ (resp. $\mathbb{M R}, \mathbb{M T}$ ).

We proceed by induction on the height of derivations. We give some representative cases below.

$(\wedge R \mathrm{c}) \quad$ Suppose that a valuation $v$ is a counterexample to the metasequent $\Sigma \Rightarrow$

$[X \succ Y, A \wedge B]$. Then in particular every sequent $\sigma \in \Sigma$ must hold on $v$, while

\footnotetext{
${ }^{9} \mathrm{An}$ anonymous referee suggests that any of our metasequent calculi may be able to be modified to be sound and complete w.r.t. global validity by simply altering $(I D)$ and replacing it with the initial metasequent $\sigma \Rightarrow \mathfrak{s}(\sigma)$ for $\mathfrak{s}$ a substitution. We leave it to the interested reader to pursue this suggestion further.
} 
$v \forall[X>Y, A \wedge B]$. In particular, this means that $v(A \wedge B) \in\{\perp, I\}$. As our valuation is truth-fitting this means that $v(A) \curlywedge v(B) \in\{\perp$, I $\}$ and so by the definition of $\curlywedge$ that either $v(A) \in\{\perp$, I $\}$ or $v(B) \in\{\perp$, I $\}$. From this it follows that either $v \forall[X \succ Y, A]$ (and thus $v \forall \Sigma \Rightarrow[X \succ Y, A]$ ) or $v \forall[X \succ Y, B]$ (and thus $v \| \Sigma \Rightarrow[X \succ Y, B])$, as desired.

$(\neg L \mathrm{p})$ Suppose that a valuation $v$ is a counterexample to the metasequent $\Sigma,[X, \neg A \succ Y] \Rightarrow \sigma$. Then in particular we have that $v \Vdash[X, \neg A \succ Y]$, from which it follows (in the interesting case) that $v(\neg A) \notin\{\top, I\}$. By the definition of $\sim$ this means that $\sim v(A) \in\{\perp, *\}$, and thus that $v(A) \in\{\top, *\}$, from which it follows that $[X \succ Y, A]$ holds on $v$, as desired.

(CASES) Suppose that a valuation $v$ is a counterexample to the metasequent $\Sigma,\left[X, X^{\prime} \succ Y, Y^{\prime}\right] \Rightarrow \sigma$. Then in particular $v \Vdash\left[X, X^{\prime} \succ Y, Y^{\prime}\right]$, and so either for some formula $A \in X \cup X^{\prime}$ we have $v(A) \in\{\perp, *\}$ or for some formula $B \in Y \cup Y^{\prime}$ we have $v(B) \in\{\top, *\}$. In either case our formula $A$ (resp. $B$ ) must be in either $X$ or $X^{\prime}$ (resp. $Y$ or $Y^{\prime}$ ), in which case we must have either $v \Vdash[X \succ Y]$ or $v \Vdash\left[X^{\prime} \succ Y^{\prime}\right]$, and consequently $v$ is either a counterexample to $\Sigma,[X \succ Y] \Rightarrow \sigma$ or $\Sigma,\left[X^{\prime} \succ Y^{\prime}\right] \Rightarrow \sigma$ as desired.

In order to prove completeness we will use a modification of the reduction tree method, in a similar way to how a related result is proved in [17].

Theorem 8 (Completeness) If a metasequent $\Sigma \Rightarrow \sigma$ is not derivable in $\mathbb{M} *+$ (resp. $\mathbb{M R} *+, \mathbb{M T} *+$ ) then there is a truth-fitting (resp. reflexive, transitive) tetravaluation which counterexamples it.

Proof To do this we will start with a given metasequent and conduct a root-first proof search in $\mathbb{M} *+$ (resp. $\mathbb{M R} *+$ or $\mathbb{M} T *+$ ), using a failed proof search in order to construct a tetravaluation $v$ which counterexamples the metasequent with which we began.

Suppose that we have an enumeration $A_{0}, A_{1}, A_{n}, \ldots$ of all the formulas in $\mathcal{L}$ in which each formula $A_{i}$ appears infinitely many times. Let $\Sigma \Rightarrow \sigma$ be a metasequent, and let us construct a tree of metasequents as follows.

- $\quad$ Let the root of our reduction tree be the metasequent $\Sigma \Rightarrow \sigma$.

- At any stage a branch of our tree is closed iff its leaf sequents are of the form $\Theta, \sigma^{\prime} \Rightarrow \theta$ and $\sigma^{\prime} \sqsubseteq \theta$ (in which case we can derive this metasequent by applying $(D i l)$ and $(D I L)$ to an instance of $(I D))$, or in the case where we are dealing with $\mathbb{M R} *+$ when our metasequent is of the form $\Sigma \Rightarrow[X \succ Y]$ where $X \cap Y \neq \varnothing$ (in which case it is the result of applying ( $D i l$ ) to an instance of $(I d))$. A branch that is not closed is open.

- For each open branch we expand it in one of two ways.

- We can root-first apply one of the conclusion active introduction rules of our calculus to the conclusion metasequent of an open leaf of our tree. So, for example, if our metasequent is of the form $\Sigma \Rightarrow[X \succ Y, A \wedge B]$ 
then we expand our branch by applying the rule $(\wedge R)_{c}$ root-first, giving us the following

$$
\frac{\Sigma \Rightarrow[X \succ Y, A \wedge B, A] \quad \Sigma \Rightarrow[X \succ Y, A \wedge B, B]}{\Sigma \Rightarrow[X \succ Y, A \wedge B]}(\wedge R)_{c}
$$

after which the leaves of this branch are $\Sigma \Rightarrow[X>Y, A \wedge B, A]$ and $\Sigma \Rightarrow[X \succ Y, A \wedge B, B]$.

- We can root-first apply one of the premise active introduction rules to a sequent $\theta_{i} \in \Theta$ where $\Theta \Rightarrow \theta$ is an open leaf metasequent of our tree. So, for example, if our leaf metasequent is of the form $\Sigma,[X \succ Y, A \wedge B] \Rightarrow \sigma$ we expand our branch by applying the rule $(\wedge R)_{p}$ root-first, giving us the following

$$
\frac{\Sigma,[X \succ Y, A \wedge B],[X \succ Y, A \wedge B, A],[X \succ Y, A \wedge B, B] \Rightarrow \sigma}{\Sigma,[X \succ Y, A \wedge B] \Rightarrow \sigma}(\wedge R)_{p}
$$

- $\quad$ (In $\mathbb{M} T *$ ) once we can no longer apply our Operational Rules we expand our branch by applying the context sharing version of the $(\mathrm{Cut})$ rule root-first to every open left in the tree, the cut-formula being the next formula in our enumeration of formulas in $\mathcal{L}$. That is to say, if $\Theta \Rightarrow[X \succ Y]$ is our leaf metasequent, and $A_{i}$ the next formula in our enumeration, then we expand that branch of our tree of metasequents as follows

$$
\frac{\Theta \Rightarrow\left[X \succ Y, A_{i}\right] \Theta \Rightarrow\left[A_{i}, X \succ Y\right]}{\Theta \Rightarrow[X \succ Y]}
$$

where our new leaf metasequents are $\Theta \Rightarrow\left[X \succ Y, A_{i}\right]$ and $\Theta \Rightarrow\left[A_{i}, X \succ Y\right]$. At this point we return, again, to applying our Operational Rules.

If we repeatedly apply the above procedure (guided, for example, by our enumeration of formulas in the language) we will either end up with a finite tree of metasequents, which can be converted into a derivation in $\mathbb{M} *+$ through applications of our external and internal structural rules, or we will have an infinite tree of metasequents. Due to the way we have constructed our tree of metasequents, if we have an infinite tree there must be an infinite branch. Let $\Sigma_{0} \Rightarrow \sigma_{0}, \Sigma_{1} \Rightarrow$ $\sigma_{1}, \Sigma_{2} \Rightarrow \sigma_{2}, \ldots$ be an enumeration of the metasequents which appear on a chosen infinite branch of our tree, with $\Sigma_{0} \Rightarrow \sigma_{0}$ being our intitial metasequent $\Sigma \Rightarrow \sigma$. Call this enumeration Red. Let $L=\left\{A \mid\right.$ For some $\left.i: \Sigma_{i} \Rightarrow[A, \Gamma \succ \Delta] \in \operatorname{Red}\right\}$ and $R=\left\{A \mid\right.$ For some $\left.i: \Sigma_{i} \Rightarrow[\Gamma \succ \Delta, A] \in \operatorname{Red}\right\}$. Consider, now, the sequent $e=[L \succ R]$, let $v_{e}$ be its exact counterexample as given in Definition 2 .

It is clear that $v_{e}$ is a tetravaluation, so all that remains to be demonstrated is that it is (1) a counterexample to the metasequent $\Sigma \Rightarrow \sigma$, (2) that it is truth-fitting, and (3) that it is reflexive if we are in $\mathbb{M R} *+$ and transitive if we are in $\mathbb{M T} *+$.

Counterexample For $v_{e}$ to be a counterexample to $\Sigma \Rightarrow \sigma$ we must have (i) $v_{e} \Vdash s$ for all $s \in \Sigma$, and (ii) $v_{e} \nvdash \sigma$. By the construction of our reduction tree it is clear that $\sigma \sqsubseteq e$, and so by Proposition 1 that $v_{e} \forall \sigma$. So it is clear that (ii) holds. For (i) suppose for reductio that $v_{c} \Downarrow s$ for some $s \in \Sigma$. Then, again by Proposition 1 we must have $s \sqsubseteq e$. By the construction of our reduction tree, again, it is easy to see 
that every branch of the tree is 'conclusion monotone' in the sense that if $i<j$ and $\Sigma_{i} \Rightarrow \sigma_{i}$ and $\Sigma_{j} \Rightarrow \sigma_{j}$ are both in Red then $\sigma_{i} \sqsubseteq \sigma_{j}$. So as $s$ is a sequent, and thus finite, it follows that we must have $s \sqsubseteq \sigma_{j}$ for some $j$. But as our reduction tree is 'premise monotone' in the sense that if $i<j$ and $\Sigma_{i} \Rightarrow \sigma_{i}$ and $\Sigma_{j} \Rightarrow \sigma_{j}$ are both in $\operatorname{Red}$ then $\Sigma_{i} \subseteq \Sigma_{j}$, it follows that we must have $s \in \Sigma_{j}$, from which it follows that our branch must be closed, which it is not. So by reductio it follows that $v_{e} \Vdash s$ for all $s \in \Sigma$.

Truth-Fittingness For this valuation to be truth-fitting we need to check that $v_{e}(A \wedge$ $B)=v_{e}(A) \curlywedge v_{e}(B)$ and $v_{e}(\neg A)=\sim v_{e}(A)$. We reason by cases.

$v_{e}(A \wedge B)=\top: \quad$ If $v_{e}(A \wedge B)=\top$ then by the definition of $v_{e}$ it follows that $A \wedge B \in L$ and $A \wedge B \notin R$. As $A \wedge B \in L$ it follows by $(\wedge L)$ that $A \in L$ and $B \in L$. As $A \wedge B \notin R$ it follows by $(\wedge R) \uparrow$ that $A \notin R$ and $B \notin R$, and thus that $v_{e}(A)=\top$ and $v_{e}(B)=\top$, and consequently that $v_{e}(A \wedge B)=v_{e}(A) \curlywedge v_{e}(B)$.

$v_{e}(A \wedge B)=\perp$ : $\quad$ If $v_{e}(A \wedge B)=\perp$ then by the definition of $v_{e}$ it follows that $A \wedge B \notin L$ and $A \wedge B \in R$. As $A \wedge B \in R$ it follows that either $A \in R$ or $B \in R$. Suppose w.l.o.g. that $A \in R$, and thus that $v_{e}(A) \in\{\perp$, I $\}$. As $A \wedge B \notin L$ it follows that either $A \notin L$ or $B \notin L$. If $A \notin L$ then $v_{e}(A)=\perp$, and thus $v_{e}(A) \curlywedge v_{e}(B)=v_{e}(A \wedge B)=\perp$ as desired. If $A \in L$ and $B \notin L$ then $v_{e}(A)=I$ and $v_{e}(B) \in\{\perp, *\}$, and thus as $I \curlywedge \perp=I \curlywedge *=\perp$, again, $v_{e}(A) \curlywedge v_{e}(B)=$ $v_{e}(A \wedge B)$ as desired.

$v_{e}(A \wedge B)=I \quad$ If $v_{e}(A \wedge B)=I$ then $A \wedge B \in L$ and $A \wedge B \in R$. As $A \wedge B \in L$ it follows by $(\wedge L)$ that $A \in L$ and $B \in L$. As $A \wedge B \in R$ it follows that either $A \in R$ or $B \in R$. From this it follows that either $v_{e}(A)=I$ or $v_{e}(B)=I$, and in either case that $v_{e}(A) \curlywedge v_{e}(B) \in\{I, \perp\}$. But for it to be the case that $v_{e}(A) \curlywedge v_{e}(B)=\perp$ we must have either $v_{e}(A)=\perp$ or $v_{e}(B)=\perp$, which we cannot have as this requires that $A \notin L$ (resp. $B \notin L$ ) and $A$ and $B$ are both in $L$, and so $v_{e}(A \wedge B)=v_{e}(A) \curlywedge v_{e}(B)=I$

$v_{e}(A \wedge B)=* \quad$ If $v_{e}(A \wedge B)=*$ then $A \wedge B \notin L$ and $A \wedge B \notin R$. As $A \wedge B \notin L$ it follows by $(\wedge L)$ that either $A \notin L$ or $B \notin L$. As $A \wedge B \notin R$ it follows by $(\wedge R) \uparrow$ that both $A \notin R$ and $B \notin R$. Thus either $v_{e}(A)=*$ or $v_{e}(B)=*$, and thus in either case that $v_{e}(A) \curlywedge v_{e}(B) \in\{*, \perp\}$. But as neither $A$ nor $B$ are in $R$ it cannot be that either $v_{e}(A)=\perp$ or $v_{e}(B)=\perp$, and so $v_{e}(A \wedge B)=v_{e}(A) \curlywedge v_{e}(B)=*$

The cases for the other connectives follow similarly.

Reflexivity and Transitivity All that remains to show is that the valuation induced by the above procedure is of the right kind, namely that if we are working with $\mathbb{M R} *$ that it is a truth-fitting reflexive tetravaluation, and that if we are working with $\mathbb{M} T *$ that it is a truth-fitting transitive tetravaluation. If we are in MT then any open leaf metasequent must have $L \cup R=\mathcal{L}$ due to the repeated root-first applications of $(\mathrm{Cut})$. If we are in $\mathbb{M R}$ then we will never have an open leaf metasequent where $L \cap R \neq \emptyset$, as such branches are closed in the presence of $(I d)$. So it is clear by the definition of $v_{e}$ that in both cases we must have $v_{e} \in \mathfrak{V}^{t}$ and $v_{e} \in \mathfrak{V}^{r}$, respectively. 
It is worth remarking on the fact that the above results also tell us that the external structural rule of $(C U T)$ is admissible in $\mathbb{M} *+, \mathbb{M R} *+$, and $\mathbb{M T} *+$.

Theorem 9 The rule of $(C U T)$ is admissible in $\mathbb{M} *+, \mathbb{M R} *+$, and $\mathbb{M T} *+$.

Proof Let $S$ be one of $\mathbb{M}, \mathbb{M R}$, or $\mathbb{M T}$. Suppose that we had a metasequent $\Sigma \Rightarrow \sigma$ such that we had (i) $\vdash_{S} \quad \Sigma \Rightarrow \sigma$ but (ii) $\nvdash_{S *+} \quad \Sigma \Rightarrow \sigma$. By (i) and Theorem 7 it follows that $\Sigma \Rightarrow \sigma$ holds on all tetravaluations/all reflexive tetravaluations/all transitive tetravaluations depending on whether $S$ is $\mathbb{M}, \mathbb{M R}$, or $\mathbb{M T}$. But by (ii) and Theorem 8 it follows that there is some tetravaluation/reflexive tetravaluation/transitive tetravaluation which is a counterexample to $\Sigma \Rightarrow \sigma$, resulting in a contradiction. So it follows that any sequent which is derivable in $S$ (and thus derivable using $(C U T)$ ) is derivable in $S *+$ (and thus derivable without using $(C U T))$.

Similarly, we also have

Theorem 10 The rule of $(C A S E S)$ is admissible in $\mathbb{M} *+, \mathbb{M R} *+$, and $\mathbb{M} T *+$.

Interestingly, the above results can also be extended to the extensions of $\mathbb{M} *+$, $\mathbb{M R} *+$, and $\mathbb{M T} *+$ by the elimination operational rules.

Proposition 2 If a metasequent $\Sigma \Rightarrow \sigma$ is derivable in $\mathbb{M} *+$ (resp. $\mathbb{M R} *+, \mathbb{M T} *+)$ then it is derivable in $\mathbb{M} p E c E *+($ resp. $\mathbb{M} p E c E R *+, \mathbb{M} p E c E T *+)$

Proof We treat the case of $\mathbb{M} *+$ and $\mathbb{M} p E c E *+$ for simplicity (the other cases are structurally similar). Firstly note that by Theorem 5 and 4 that as the premise and conclusion elimination rules are derivable in $\mathbb{M}=\operatorname{SpIcI}$ that (i) $\Sigma \Rightarrow \sigma$ is derivable in $\mathbb{M}$ iff it is derivable in $\mathbb{M} p E c E$. But by Theorem 9 and 10 it follows that (ii) $\Sigma \Rightarrow \sigma$ is derivable in $\mathbb{M}$ iff it is derivable in $\mathbb{M} *+$. So it follows that $\Sigma \Rightarrow \sigma$ is derivable in $\mathbb{M} *+$ iff by (ii) it is derivable in $\mathbb{M}$ iff by (i) it is derivable in $\mathbb{M} p E c E$.

\section{Reducing Metainferences to Inferences}

As mentioned in the introduction, much of the philosophical interest in metainferences has centered around the fact that the metainferential consequence relations determined by nontransitive logics can be embedded into the SET-FMLA consequence relations for paraconsistent logics. In particular [2] and [6] have shown that a metainference is valid according to the nontransitive logic ST (essentially derivable in our $S c I c E R$ ) iff its translation is valid according to the paraconsistent logic $\mathbf{L P}$, on the basis of which they have claimed that (contra [26]) that ST is not classical logic, 
with the authors of [6] going as far as to claim that it is in fact LP. ${ }^{10}$ Similarly, in [13] it is conjectured that the logic $L K R^{+}$there (whose metainferences are those of our $S c I c E T$ ) can be faithfully embedded into Strong Kleene logic. All of these arguments have been semantic in nature and, like most work on metainferences, were conducted at the level of inferences rather than metainferences. What we will do in this section is to bring some uniformity to this discussion, providing purely syntactic proofs of these results by making use of our metasequent calculi.

Before moving on, though, it is worthwhile to place this discussion withing the broader context of the existing formal and philosophical literature on translations between logics. One aspect which is worth highlighting is the way in which the situation here differs from usual treatments of translations between logics, as these usually involve functions between objects of the same syntactic category (viz. formulas), and typically exhibit a connection between logics couched within the same logical framework, while the translations which we will be dealing with here are ones which will map between objects in different syntactic categories in order to relate logics in different logical frameworks. ${ }^{11}$ In particular, given a function $f$ which maps sequents from $\mathfrak{A}$ to formulas of $\mathcal{L}$, and a function $s$ which maps formulas of $\mathcal{L}$ to sequents from $\mathfrak{A}$, let us say that the metainferential consequence relation $\Rightarrow_{1}$ can be reduced to the logic $\vdash_{2}$ just in case:

$$
\begin{aligned}
& \text { (i) } \sigma_{1}, \ldots, \sigma_{n} \Rightarrow_{1} \sigma \text { only if } f\left(\sigma_{1}\right), \ldots, f\left(\sigma_{n}\right) \vdash_{2} f(\sigma) \\
& \text { (ii) } A_{1}, \ldots, A_{n} \vdash_{2} B \text { only if } s\left(A_{1}\right), \ldots, s\left(A_{n}\right) \Rightarrow_{1} s(B) \\
& \text { (iii) } A \dashv \vdash_{2} f(s(A)) \text {. }
\end{aligned}
$$

That is to say, the metainferential consequence relation $\Rightarrow_{1}$ can be reduced to $\vdash_{2}$ whenever, considered as consequence relations, they are translationally equivalent. In particular we will be concerned with the following two translations $\tau$ and $\rho:{ }^{12}$

$$
\rho(A)=[\succ A] \quad \tau([\Gamma \succ \Delta])= \begin{cases}\neg(\bigwedge \Gamma) \vee(\bigvee \Delta) & \Gamma \neq \emptyset, \Delta \neq \emptyset \\ \bigvee \Delta & \Gamma=\emptyset, \Delta \neq \emptyset \\ \neg \bigwedge \Gamma & \Gamma \neq \emptyset, \Delta=\emptyset \\ \mathbf{F} & \Gamma=\emptyset, \Delta=\emptyset\end{cases}
$$

With these two translations in hand we will now move on to show how various metainferential consequence relations can be reduced to standard paraconsistent and paracomplete logics. To do this we will need to have a sequent calculus presentation of the paraconsistent and paracomplete logics we will be working with, and it is to this which we will now turn.

\footnotetext{
${ }^{10}$ An earlier antecedent to these results can be seen in [25].

${ }^{11}$ For more information on translations between logics see [12, pp.12-38] and [29]. The present setting is one in which the more abstract approach advocated for in [29] is definitely useful, where logics are just arbitrary closure operations on sets and translations are just continuous maps between logics. It bears noting that the translations which we will be concerned with here are all conservative in the sense of [9] (in fact essentially showing that various metainferential logics are translationally equivalent to various consequence relations, for more on which see chapter 5 of [12]).

${ }^{12}$ In what follows we will gloss over the case where $\Gamma=\Delta=\emptyset$ for the sake of simplicity.
} 


\subsection{A Sequent Calculus for FDE and related logics}

Our sequent calculi for $F D E, \mathbf{K 3}$ and $\mathbf{L P}$ are similar to the one given in [24] for $F D E$, although in this case we will make use of a single, as opposed to multipleconclusion, formulation. We will begin by defining a sequent calculus for $F D E$, and then show how to extend this to a sequent calculus for K3 and LP. Our sequent calculi for $F D E$ and its extensions manipulate sequents $\langle\Gamma, A\rangle$ where $\Gamma \subseteq \mathcal{L}$ and $A \in \mathcal{L}$, which we will write henceforth as $\Gamma \Vdash A$.

Our sequent calculus $\mathcal{S}_{F D E}$ has as structural rules $[\mathrm{Id}]$ and [Cut].

$$
\overline{\Gamma, A \Vdash A}_{[I d]}^{\left[I \Vdash A \quad A, \Gamma^{\prime} \Vdash C\right.}\left[{ }_{[C u t]}\right]
$$

The system's operational rules are split into 'positive' and 'negative' versions. The system has the following positive operational rules.

$$
\begin{gathered}
\frac{\Gamma, A, B \Vdash C}{\Gamma, A \wedge B \Vdash C}[\wedge L] \quad \frac{\Gamma \Vdash A \quad \Gamma \Vdash B}{\Gamma \Vdash A \wedge B}[\wedge R] \\
\frac{\Gamma, A \Vdash C \quad \Gamma, B \Vdash C}{\Gamma, A \vee B \Vdash C}[\vee L] \\
\frac{\Gamma \Vdash A}{\Gamma \Vdash A \vee B}\left[\vee R_{]} \quad \frac{\Gamma \Vdash B}{\Gamma \Vdash A \vee B}\left[\vee R_{2}\right]\right.
\end{gathered}
$$

The system's negative operational rules are then the following.

$$
\begin{gathered}
\frac{\Gamma, \neg A \Vdash C \quad \Gamma, \neg B \Vdash C}{\Gamma, \neg(A \wedge B) \Vdash C}[\neg \wedge L] \quad \frac{\Gamma \Vdash \neg A}{\Gamma \Vdash \neg(A \wedge B)}\left[\neg \wedge R_{]} \quad \frac{\Gamma \Vdash \neg B}{\Gamma \Vdash \neg(A \wedge B)}\left[\neg \wedge R_{2}\right]\right. \\
\frac{\Gamma, \neg A, \neg B \Vdash C}{\Gamma, \neg(A \vee B) \Vdash C}[\neg \vee L] \quad \frac{\Gamma \Vdash \neg A \quad \Gamma \Vdash \neg B}{\Gamma, \Vdash \neg(A \vee B)}[\neg \vee R]
\end{gathered}
$$

Finally we also have the rules for double negation.

$$
\frac{\Gamma \Vdash A}{\Gamma \Vdash \neg \neg A}[\neg \neg L] \quad \frac{\Gamma, A \Vdash C}{\Gamma, \neg \neg A \Vdash C}[\neg \neg R]
$$

It is easy to see that this is a sound and complete sequent calculus for $F D E$. In order to extend it to a calculus which is sound and complete for $\mathbf{K 3}$ we simply add the rule [EFQ], and for one which is sound and complete for $\mathbf{L P}$ with the rule [EXM].

$$
\begin{gathered}
\overline{\Gamma \Vdash A \vee \neg A}[E X M] \\
\frac{\Gamma \Vdash A \quad \Gamma \Vdash \neg A}{\Gamma \Vdash C}[E F Q]
\end{gathered}
$$

One useful syntactic Lemma which we will make extensive use of below is the following.

Lemma 1 Suppose that $A \Vdash B$. Then if $\Gamma \Vdash C_{1} \vee \ldots C_{n} \vee A$ then $\Gamma \Vdash C_{1} \vee \ldots C_{n} \vee$ $B$.

\subsection{The Translations}

What we will now show is that $\mathbb{M}, \mathbb{M} T$ and $\mathbb{M R}$ are translationally equivalent to $F D E, K 3$ and $L P$, respectively. 
Theorem 11 For all sequents $\sigma_{1}, \ldots, \sigma_{n}, \sigma$ we have the following:

1. $\sigma_{1}, \ldots, \sigma_{n} \Rightarrow \sigma$ is derivable in $\mathbb{M}$ only if $\tau\left(\sigma_{1}\right), \ldots, \tau\left(\sigma_{n}\right) \vdash_{F D E} \tau(\sigma)$.

2. $\sigma_{1}, \ldots, \sigma_{n} \Rightarrow \sigma$ is derivable in $\mathbb{M T}$ only if $\tau\left(\sigma_{1}\right), \ldots, \tau\left(\sigma_{n}\right) \vdash_{K 3} \tau(\sigma)$.

3. $\sigma_{1}, \ldots, \sigma_{n} \Rightarrow \sigma$ is derivable in $\mathbb{M R}$ only if $\tau\left(\sigma_{1}\right), \ldots, \tau\left(\sigma_{n}\right) \vdash_{L P} \tau(\sigma)$.

Proof The proof here is by induction on the height of derivations in the appropriate extension of $\mathbb{M}$. We give some representative cases to give a feel for what's going on.

$(\wedge L) c \quad$ Suppose that we have a derivation of height $n$ which ends with an application of $(\wedge L) c$ as follows.

$$
\frac{\Sigma \Rightarrow[X, A, B \succ Y]}{\Sigma \Rightarrow[X, A \wedge B \succ Y]}(\wedge L) c
$$

By the induction hypothesis we have that $\tau(\Sigma) \Vdash \neg X \vee \neg A \vee \neg B \vee Y$. Rewriting $X \vee \neg A \vee \neg B \vee Y$ as $(X \vee Y) \vee(\neg A \vee \neg B)$. Noting that $\neg A \vee \neg B \Vdash \neg(A \wedge B)$, we can then appeal to Lemma 1 to conclude that $\tau(\Sigma) \Vdash \neg X \vee \neg(A \wedge B) \vee Y$, as desired.

(Cut) Suppose that we are in $\mathbb{M T}$ have a derivation of height $n$ which ends in an application of $(\mathrm{Cut})$ as follows.

$$
\frac{\Sigma \Rightarrow[X \succ Y, A] \quad \Sigma \Rightarrow\left[A, X^{\prime} \succ Y^{\prime}\right]}{\Sigma \Rightarrow\left[X, X^{\prime} \succ Y, Y^{\prime}\right]}(\text { Cut })
$$

By the induction hypothesis we have that $\tau(\Sigma) \Vdash \neg X \vee Y \vee A$ and $\tau(\Sigma) \Vdash \neg A \vee$ $\neg X \vee Y$. By $[\vee R]$ on both premises it follows that: $\tau(\Sigma) \Vdash \neg X \vee \neg X^{\prime} \vee Y \vee Y^{\prime} \vee A$ and $\tau(\Sigma) \Vdash \neg A \vee \neg X \vee \neg X^{\prime} \vee Y \vee Y^{\prime}$. Applying $[\wedge R]$ to both sequents, and abbreviating $\neg X \vee \neg X^{\prime} \vee Y \vee Y^{\prime}$ as $S$ we have that $\tau(\Sigma) \Vdash(S \vee A) \wedge(S \vee \neg A)$. We can then apply [Cut] to this and the $K$ 3-provable sequent $(S \vee A) \wedge(S \vee \neg A) \Vdash$ $S \vee(A \wedge \neg A)$, and then finally appealing to the $K$ 3-provable sequent $A \wedge \neg A \Vdash S$ conclude $\tau(\Sigma) \Vdash S \vee S$ which by a further single application of [Cut] on the sequent $S \vee S \Vdash S$ yields the desired result.

(Id) Suppose that we are in MR and we have a derivation of height 1 of an instance of $(I d)$. The translation of the resulting sequent is an instance of [EXM], and so the result follows.

$(C A S E S)$ Suppose we have a derivation of height $n$ which ends in an application of $(C A S E S)$ as follows.

$$
\frac{\Sigma,[X \succ Y] \Rightarrow \sigma \quad \Sigma,\left[X^{\prime}>Y^{\prime}\right] \Rightarrow \sigma}{\Sigma,\left[X, X^{\prime} \succ Y, Y^{\prime}\right] \Rightarrow \sigma}(C A S E S)
$$

By the IH we have that $\tau(\Sigma), \neg X \vee Y \Vdash \tau(\sigma)$ and $\tau(\Sigma), \neg X^{\prime} \vee Y^{\prime} \Vdash \tau(\sigma)$, from which we can conclude $\tau(\Sigma), \neg X \vee Y \vee \neg X^{\prime} \vee Y \Vdash \tau(\sigma)$ by a single application of $[\vee L]$, which (after some reassociation) is what we're after.

Theorem 12 For all formulas $A_{1}, \ldots, A_{n}, B$ we have the following

1. $A_{1}, \ldots, A_{n} \vdash_{F D E} B$ only if $\left[\succ A_{1}\right], \ldots,\left[\succ A_{n}\right] \Rightarrow[\succ B]$ is derivable in $\mathbb{M}$ 
2. $A_{1}, \ldots, A_{n} \vdash_{K 3} B$ only if $\left[\succ A_{1}\right], \ldots,\left[\succ A_{n}\right] \Rightarrow[\succ B]$ is derivable in $\mathbb{M T}$

3. $A_{1}, \ldots, A_{n} \vdash_{L P} B$ only if $\left[\succ A_{1}\right], \ldots,\left[\succ A_{n}\right] \Rightarrow[\succ B]$ is derivable in $\mathbb{M R}$

Proof The proof is by induction on the height of derivations in the appropriate extension of $F D E$, with some representative cases given below.

$[\wedge L]$ Suppose that we have a derivation of height $n$ whose final inference is as follows:

$$
\frac{A, B, \Gamma \Vdash C}{A \wedge B, \Gamma \Vdash C}[\wedge L]
$$

By the induction hypothesis we have a proof of $[\succ A],[\succ B],\left\{\left[\succ \gamma_{i}\right] \mid \gamma_{i} \in \Gamma\right\} \Rightarrow$ $[\succ C]$ from which we can derive $[\succ A \wedge B],\left\{\left[\succ \gamma_{i}\right] \mid \gamma_{i} \in \Gamma\right\} \Rightarrow[\succ C]$ by a single application of $(\wedge R) p$.

$[\wedge R]$ Suppose that we have a derivation of height $n$ whose final inference is as follows:

$$
\frac{\Gamma \Vdash A \quad \Gamma \Vdash B}{\Gamma \Vdash A \wedge B}[\wedge R]
$$

By the induction hypothesis we have a proof of $\left\{\left[\succ \gamma_{i}\right] \mid \gamma_{i} \in \Gamma\right\} \Rightarrow[\succ A]$ and $\left\{\left[\succ \gamma_{i}\right] \mid \gamma_{i} \in \Gamma\right\} \Rightarrow[\succ B]$, from which we can derive $\left\{\left[\succ \gamma_{i}\right] \mid \gamma_{i} \in \Gamma\right\} \Rightarrow$ $[\succ A \wedge B]$ by a single application of $(\wedge R) c$.

$[\neg \wedge L]$ Suppose that we have a derivation of height $n$ whose final inference is as follows:

$$
\frac{\Gamma, \neg A \Vdash C \quad \Gamma, \neg B \Vdash C}{\Gamma, \neg(A \wedge B) \Vdash C}[\neg \wedge L]
$$

By the induction hypothesis we have a proof of $[\succ \neg A],\left\{\left[\succ \gamma_{i}\right] \mid \gamma_{i} \in \Gamma\right\} \Rightarrow$ $[\succ C]$ and $[\succ \neg B],\left\{\left[\succ \gamma_{i}\right] \mid \gamma_{i} \in \Gamma\right\} \Rightarrow[\succ C]$, and can proceed as follows.

$$
\begin{aligned}
& \frac{[\succ \neg A],\left\{\left[\succ \gamma_{i}\right] \mid \gamma_{i} \in \Gamma\right\} \Rightarrow[\succ C]}{\frac{[A \succ],\left\{\left[\succ \gamma_{i}\right] \mid \gamma_{i} \in \Gamma\right\} \Rightarrow[\succ C]}{[A, B \succ L) p \uparrow} \quad \frac{[\succ \neg B],\left\{\left[\succ \gamma_{i}\right] \mid \gamma_{i} \in \Gamma\right\} \Rightarrow[\succ C]}{[B \succ],\left\{\left[\succ \gamma_{i}\right] \mid \gamma_{i} \in \Gamma\right\} \Rightarrow[\succ C]}}(\neg L) p \uparrow \\
& \frac{[A, B \succ],\left\{\left[\succ \gamma_{i}\right] \mid \gamma_{i} \in \Gamma\right\} \Rightarrow[\succ C]}{\left[\frac{[A B \succ],\left\{\left[\succ \gamma_{i}\right] \mid \gamma_{i} \in \Gamma\right\} \Rightarrow[\succ C]}{[\succ \neg(A) p}(\neg B)\right],\left\{\left[\succ \gamma_{i}\right] \mid \gamma_{i} \in \Gamma\right\} \Rightarrow[\succ C]}(\neg R)
\end{aligned}
$$

[EFQ] Suppose that we have a proof of height $n$ which ends as follows:

$$
\frac{\Gamma \Vdash A \quad \Gamma \Vdash \neg A}{\Gamma \Vdash C}[E F Q]
$$

By the IH we have that $\left\{\left[\succ \gamma_{i}\right] \mid \gamma_{i} \in \Gamma\right\} \Rightarrow[\succ A]$ and $\left\{\left[\succ \gamma_{i}\right] \mid \gamma_{i} \in \Gamma\right\} \Rightarrow$ $[\succ \neg A]$. We proceed as follows

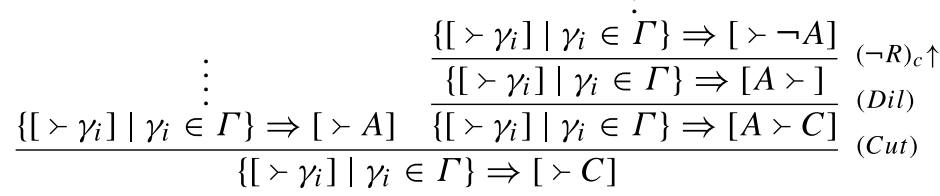

[EXM] Suppose that we have a proof of height 1 which consists of a single application of [EXM]

$$
\overline{\Gamma \Vdash A \vee \neg A}[E X M]
$$


We proceed as follows to show that the translation of this sequent is provable in MR.

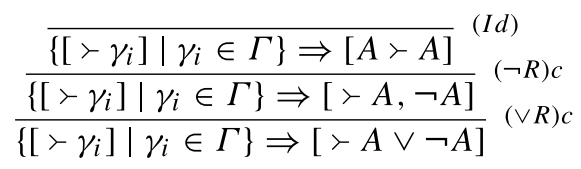

Lemma 2 For all formulas $A$ we have that

$$
\text { (i) } A \Vdash \tau(\rho(A)) \quad \text { (ii) } \tau(\rho(A)) \Vdash A
$$

Making use of this Lemma and the previous Theorem we can then conclude the following.

Theorem 13 For all sequents $\sigma_{1}, \ldots, \sigma_{n}, \sigma$ we have the following:

1. $\sigma_{1}, \ldots, \sigma_{n} \Rightarrow \sigma \in \mathbb{M}$ if and only if $\tau\left(\sigma_{1}\right), \ldots, \tau\left(\sigma_{n}\right) \vdash_{F D E} \tau(\sigma)$.

2. $\sigma_{1}, \ldots, \sigma_{n} \Rightarrow \sigma \in \mathbb{M T}$ if and only if $\tau\left(\sigma_{1}\right), \ldots, \tau\left(\sigma_{n}\right) \vdash_{K 3} \tau(\sigma)$.

3. $\sigma_{1}, \ldots, \sigma_{n} \Rightarrow \sigma \in \mathbb{M R}$ if and only if $\tau\left(\sigma_{1}\right), \ldots, \tau\left(\sigma_{n}\right) \vdash_{L P} \tau(\sigma)$.

Part 2 of both Theorem 12 and Theorem 13 settle the open question in [13, p.121] in the affirmative. We leave it to others, for the time being, to determine how we should react to these results, and in particular whether we should regard them, as does [6, p.402f] as showing that $L P$ and $S T$ (essentially out MR), and hence $K 3$ and MT are the same logic.

\section{Conclusion}

This only begins to scratch the surface of work that should be done on metasequents and the metasequent calculus. Some obvious avenues for initial investigation are the following.

- One advantage which the present proof-theoretic treatment of metainferences has over that in, for example, [5], is that we are not tied to the particular logics we have treated using tetravaluations. One obvious way of exploiting that feature of the present calculus is to investigate the prospects for giving a metasequent calculus for noncontractive logics, some initial problems which would need to be addressed here being those outlined above in connection with the (CASES) rule.

- Most of the proof-theoretic work we have done here has made heavy use of the outer structural rules, in particular $(C U T)$ and $(C A S E S)$. While we have shown semantically that these rules are admissible in metasequent calculi which lack them (Theorem 9, Theorem 10), it would be of some benefit in furthering our understanding of the metasequent calculus to have direct constructive elimination proofs for these rules. In future work I hope to peruse the full structural proof 
theory of the metasequent calculus. Doing this may also help us to see what the options are for the affine noncontractive logician mentioned in the previous bullet point.

- Finally, one of the advantages of the metasequent calculus is that it makes the outer structural rules visible. One direction for future work is to exploit this visibility in order to provide syntactic analogues of the various mixed metainferential logics presented in [22].

Let us take stock. In the present paper we have taken the first steps in regarding metasequents are bona fide logical objects, and constructed sequent calculi which allow us to reason explicitly about metasequents for a range of nonreflexive and nontransitive logics. We then used our metasequent calculi to provide syntactic proofs of various collapse results. There is a vast range of other questions which the present machinery raises and can be used to address, but we will leave that for future work.

Acknowledgements I would like to thank audiences at the VI Workshop on Philosophical Logic at the University of Buenos Aires, the Melbourne Logic Group, the Berkeley Logic Colloquium, and the Logic \& Philosophy special session at the 2021 Pacific APA. Special thanks are also due to David Ripley, Shawn Standefer, and two anonymous referees for this journal for their helpful comments, and to Eduardo Barrio and Paul Egré for their patience and understanding.

\section{Declarations}

Conflict of Interests The authors declare that they have no conflict of interest.

Open Access This article is licensed under a Creative Commons Attribution 4.0 International License, which permits use, sharing, adaptation, distribution and reproduction in any medium or format, as long as you give appropriate credit to the original author(s) and the source, provide a link to the Creative Commons licence, and indicate if changes were made. The images or other third party material in this article are included in the article's Creative Commons licence, unless indicated otherwise in a credit line to the material. If material is not included in the article's Creative Commons licence and your intended use is not permitted by statutory regulation or exceeds the permitted use, you will need to obtain permission directly from the copyright holder. To view a copy of this licence, visit http://creativecommons.org/licenses/by/4.0/.

\section{References}

1. Avron, A. (1996). The method of hypersequents in the proof theory of propositional non-classical logics. In W. Hodges, M. Hyland, C. Steinhorn, \& J. Truss (Eds.) Logic: from foundations to applications, pp 1-32. Oxford University Press, Oxford.

2. Barrio, E., Rosenblatt, L., \& Tajer, D. (2015). The Logics of Strict-Tolerant Logic. Journal of Philosophical Logic, 44, 551-571.

3. Belnap, N. D. (1977). A useful four-valued logic. In Modern Uses of Multiple-Valued Logic, pp 8-37. D. Reidel, Dordrecht.

4. Cobreros, P., Égré, P., Ripley, D., \& van Rooij, R. (2014). Reaching transparent truth. Mind, 122(488), 841-866.

5. Cobreros, P., Rosa, E. L., \& Tranchini, L. (201X). (I can't get no) antisatisfaction. Synthese, forthcoming.

6. Dicher, B., \& Paoli, F. (2019). ST, LP, and Tolerant Metainferences. In C. Başkent, \& T. M. Ferguson (Eds.) Graham Priest on Dialetheism and Paraconsistency, pp 383-407. Springer.

7. Dicher, B., \& Paoli, F. (2021). The original sin of proof-theoretic semantics. Synthese, 198, 615-640.

8. Dummett, M. (1977). Elements of intuitionism. Oxford: Oxford University Press. 
9. Feitosa, H. D. A., \& D'Ottaviano, I. M. L. (2001). Conservative Translations. Annals of Pure and Applied Logic, 108, 205-227.

10. Fjellstad, A. (2016). Naive modus ponens and failure of transitivity. Journal of Philosophical Logic, $45(1), 65-72$.

11. Fjellstad, A. (2017). Non-Classical Elegance for Sequent Calculus Enthusiasts. Studia Logica, 105, 93-119.

12. French, R. (2011). Translational embeddings in modal logic. Ph.D. Thesis, Clayton.

13. French, R. (2016). Structural Reflexivity and the Paradoxes of Self-Reference. Ergo, 3(5), 113-131.

14. French, R., \& Ripley, D. (2019). Valuations: bi, tri and tetra. Studia Logica, 107, 1313-1346.

15. French, R., \& Ripley, D. (201X). Two Traditions in Abstract Valuational Model Theory. Synthese, Forthcoming.

16. Gabbay, D. M. (1981). Semantical Investigations in Heyting's Intuitionistic Logic. Synthese Library, vol 148, D. Reidel Publishing Company, Dordrecht.

17. Hösli, B., \& Jäger, G. (1994). About Some Symmetries of Negation. Journal of Symbolic Logic, 59(2), 473-485.

18. Humberstone, L. (1988). Heterogeneous Logic. Erkenntnis, 29, 395-435.

19. Humberstone, L. (2011). The Connectives. Cambridge, Massachusetts: MIT Press.

20. Kraus, S., Lehmann, D., \& Magidor, M. (1990). Nonmonotonic Reasoning, Preferential Models and Cumulative Logics. Artificial Intelligence, 44, 167-207.

21. Negri, S. (2005). Proof Analysis in Modal Logic. Journal of Philosophical Logic, 34, 507-544.

22. Pailos, F. M. (2019). A family of metainferential logics. Journal of Applied Non-Classical Logics, 29(1), 97-120.

23. Priest, G. (1979). The Logic of Paradox. Journal of Philosophical Logic, 8, 219-241.

24. Pynko, A. P. (1995). Characterizing Belnap's Logic via De Morgan's Laws. Mathematical Logic Quarterly, 41, 442-454.

25. Pynko, A. P. (2010). Gentzen's Cut-Free Calculus Versus the Logic of Paradox. Bulletin of the Section of Logic, 39(1/2), 35-42.

26. Ripley, D. (2012). Conservatively Extending Classical Logic with Transparent Truth. Review of Symbolic Logic, 5(2), 354-378.

27. Ripley, D. (2015). Comparing Substructural Theories of Truth. Ergo, 2, 299-328.

28. Ripley, D. (2015). Contraction and Closure. Thought, 4, 131-138.

29. Silva, J. J. D., D’Ottaviano, I. M. L., \& Sette, A. M. (1999). Translations Between Logics. In X. Caicedo, \& C.H. Montenegro (Eds.) Models, Algebras and Proofs, pp 435-448. Marcel Dekker, New York.

30. Singh, D., Ibrahim, A. M., Yohanna, T., \& Singh, J. N. (2008). A Systematization of Fundamentals of Multisets. Lecturas Matemáticas, 29, 33-48.

31. Teijeiro, P. (201X). Strength and Stability. Unpublished.

Publisher's Note Springer Nature remains neutral with regard to jurisdictional claims in published maps and institutional affiliations. 